Claremont Colleges

Scholarship@ Claremont

All HMC Faculty Publications and Research

HMC Faculty Scholarship

$11-1-1980$

\title{
Existence and Uniqueness for a Variational Hyperbolic System without Resonance
}

Peter W. Bates

Texas A \& M University - College Station

Alfonso Castro

Harvey Mudd College

\section{Recommended Citation}

Bates, Peter W. and Alfonso Castro. "Existence and uniqueness for a variational hyperbolic system without resonance" Nonlinear Analysis TMA, Vol. 4, No. 6(1980), pp. 1151-1156.

This Article - postprint is brought to you for free and open access by the HMC Faculty Scholarship at Scholarship @ Claremont. It has been accepted for inclusion in All HMC Faculty Publications and Research by an authorized administrator of Scholarship @ Claremont. For more information, please contact scholarship@cuc.claremont.edu. 


\title{
EXISTENCE AND UNIQUENESS FOR A VARIATIONAL HYPERBOLIC SYSTEM WITHOUT RESONANCE
}

\author{
Peter W. Bates* and Alfonso Castro ${ }^{\dagger}$
}

* Texas A\&M University, College Station, Texas 77843, U.S.A.; and † Centro de Investigacion del IPN, Apartado Postal 14-740, Mexico D.F.

(Received 21 May 1979; revised 15 October 1979)

Key Words and Phrases: Nonlinear wave equation, system, minimax, critical point, Galerkin approximation, weak solution, boundary value problem.

\section{INTRODUCTION}

IN THIS PAPER we study the existence of weak solutions of the problem

$$
\begin{cases}\square u+\nabla G(u)=f(t, x) & (t, x) \in \Omega \equiv(0, \pi) \times(0, \pi) \\ u(t, x)=0 & (t, x) \in \partial \Omega,\end{cases}
$$

where $\square$ is the wave operator $\partial^{2} / \partial t^{2}-\partial^{2} / \partial x^{2}, G: \mathbf{R}^{n} \rightarrow \mathbf{R}$ is a function of class $C^{2}$ such that $\nabla G(0)=0$ and $f: \bar{\Omega} \rightarrow \mathbf{R}^{n}$ is a continuous function having first derivative with respect to $t$ in $\left(L_{2}(\Omega)\right)^{n}$ and satisfying

$$
f(0, x)=f(\pi, x)=0
$$

for all $x \in[0, \pi]$.

We assume that there exist two $n \times n$ real symmetric matrices $A \leqslant B$ with eigenvalues $\alpha_{1} \leqslant \alpha_{2} \leqslant \ldots \leqslant \alpha_{n}$ and $\beta_{1} \leqslant \beta_{2} \leqslant \ldots \leqslant \beta_{n}$ respectively, such that

$$
\left(\bigcup_{i=1}^{n}\left[\alpha_{i}, \beta_{i}\right]\right) \cap\left\{k^{2}-j^{2} ; k, j \in \mathbf{N}\right\}=\varnothing
$$

and

$$
A \leqslant\left(\partial^{2} G(u) / \partial u_{i} \partial u_{j}\right) \leqslant B \quad \text { for all } u \in \mathbf{R}^{n}
$$

Our main result is

THEOREM A. If (1.3) and (1.4) hold then (1.1) has a unique weak solution. In addition, such a weak solution belongs to $\left(\dot{H}^{1}(\Omega)\right)^{n}$.

A result analogous to Theorem A was proved by Ahmad [1] (existence) and Lazer [2] (uniqueness) for a second order system of ODEs.

Our interest in proving Theorem A came from noticing that a simple extension of the results in [4] to systems, shows that (1.1) has a unique weak solution if there exist two real numbers $p$ 
and $q$ such that $[p, q] \cap\left\{k^{2}-j^{2}: k, j \in \mathbf{N}\right\}=\varnothing$ and

$$
p I \leqslant\left(\partial^{2} G(u) / \partial u_{i} \partial u_{j}\right) \leqslant q I \quad \text { for all } u \in \mathbf{R}^{n}
$$

Unfortunately, the methods of [4] do not seem to extend to cover the case when we assume (1.4) rather than $\left(1.4^{\prime}\right)$.

Let us denote by $R(\square)$ and $\operatorname{Ker}(\square)$ the range and kernal respectively of the operator $\square: D(\square) \subset\left(L^{2}(\Omega)\right)^{n} \rightarrow\left(L_{2}(\Omega)\right)^{n}$ with Dirichlet boundary condition. We prove Theorem A using a Galerkin approximation procedure. At each finite dimensional step we prove the existence of an approximate solution by applying a minimax theorem due to Lazer-Landesman-Meyers [3]. Condition (1.4) allows us to give an a priori estimate in $\left(L_{2}(\Omega)\right)^{n}$ for the approximate solutions. The fact that the operator $\square$ with Dirichlet boundary condition has a compact inverse on $R(\square)$ gives us the existence of $u \in R(\square)$ and $v \in \operatorname{Ker}(\square)$ so that $u+v$ satisfies (1.1) in a weak sense.

The methods used here apply to (1.1) with other boundary conditions (Neumann, periodic, mixed) with very little modification.

Finally we remark that if condition (1.4) is replaced by: there exists $r>0$ such that

$$
A \leqslant\left(\partial^{2} G(u) / \partial u_{i} \partial u_{j}\right) \leqslant B \quad \text { for } \quad\|u\| \geqslant r
$$

then it can be proved that (1.1) has a solution. This solution is not necessarily unique and in $\left(H^{1}(\Omega)\right)^{n}$. Assuming (1.4) rather than $\left(1.4^{\prime \prime}\right)$ gives us the advantage of obtaining a much simpler variational characterization of the approximate solutions which has numerical analytic implications (see [3, Section 7]).

\section{NOTATIONS AND PREIIMINARY I.FMMAS}

We let $\left\{a_{i} ; i=1, \ldots, n\right\}$ and $\left\{b_{i} ; i=1, \ldots, n\right\}$ be orthonormal bases of $\mathbf{R}^{n}$ such that

$$
A a_{i}=\alpha_{i} a_{i} \quad B b_{i}=\beta_{i} b_{i} \quad \text { for } \quad i=1, \ldots, n .
$$

We denote by $\phi_{k l}: \bar{\Omega} \rightarrow \mathbf{R}$ the function defined by $\phi_{k l}(t, x)=(2 / \pi) \sin (k t) \sin (l x)$. Clearly $\left\{\phi_{k l} ; k, l \in \mathbf{N}\right\}$ is a complete orthonormal set in $L_{2}(\Omega)$. Moreover, $\left\{\phi_{k k} ; k=1,2, \ldots\right\}$ is a complete orthonormal set in $\operatorname{Ker}(\square)$.

For each positive integer $N$ we define

$$
\begin{gathered}
X_{N}=\left\{\sum_{i, k, l} \mu_{i k l} \phi_{k l} b_{i} ; 1 \leqslant i \leqslant n, k^{2}-l^{2}>\beta_{i},\left|k^{2}-l^{2}\right| \leqslant N, k^{2} \leqslant N, \mu_{i k l} \in \mathbf{R}\right\}, \\
Y_{N}=\left\{\sum_{i, k, l} \mu_{i k l} \phi_{k l} b_{i} ; 1 \leqslant i \leqslant n, k^{2}-l^{2}<\beta_{i},\left|k^{2}-l^{2}\right| \leqslant N, k^{2} \leqslant N, \mu_{i k l} \in \mathbf{R}\right\}, \\
Z_{N}=\left\{\sum_{i, k, l} \mu_{i k l} \phi_{k l} a_{i}: 1 \leqslant i \leqslant n, k^{2}-l^{2}<\alpha_{i},\left|k^{2}-l^{2}\right| \leqslant N, k^{2} \leqslant N, \mu_{i k l} \in \mathbf{R}\right\},
\end{gathered}
$$

and

$$
E_{N}=\left\{\sum_{k, l} \phi_{k l} c_{k l} ;\left|k^{2}-l^{2}\right| \leqslant N, k^{2} \leqslant N, c_{k l} \in \mathbf{R}^{n}\right\}
$$


Clearly $X_{N} \oplus Y_{N}=E_{N}$ and $\bigcup_{N=1}^{\infty} E_{N}$ is dense in $\left(L_{2}(\Omega)\right)^{n}$. We let $\langle,\rangle_{0}$ and \|\|$_{0}$ denote the usual inner product and norm in $\left(L_{2}(\Omega)\right)^{n}$, respectively, and let \|\|$_{1}$ denote the norm in $\left(\dot{H}^{1}(\Omega)\right)^{n}$ given by $\|v\|_{1}^{2}=\int_{\Omega}\left(|\partial v / \partial t|^{2}+|\partial v / \partial x|^{2}\right)$.

We will need the main result of [3] restated here as:

LemMa 2.1. Let $j$ be a $C^{2}$ functional on a Hilbert space $H$. Suppose $X$ and $Z$ are closed subspaces of $H$ (not necessarily orthogonal) so that $X$ is finite dimensional and $H=X \oplus Z$. If there exist constants $m_{1}, m_{2}>0$ such that

$$
\begin{aligned}
& \left\langle D^{2} j(u) w, w\right\rangle \geqslant m_{1}\|w\|^{2} \quad \text { and } \\
& \left\langle D^{2} j(u) v, v\right\rangle \leqslant-m_{2}\|v\|^{2} \quad \text { for all } u \in H, w \in Z, v \in X,
\end{aligned}
$$

then there exists a unique $u_{0} \in H$ such that $\nabla j\left(u_{0}\right)=0$ and $j\left(u_{0}\right)=\max _{x \in X} \min _{y \in Z} j(x+y)$.

Define the functional $J:\left(\stackrel{\circ}{H^{1}}(\Omega)\right)^{n} \rightarrow \mathbf{R}$ by

$$
J(u)=\int_{\Omega}[(\partial u / \partial x, \partial u / \partial x)-(\partial u / \partial t, \partial u / \partial t)] / 2+G(u)-(f, u) .
$$

where (, ) denotes the usual inner product in $\mathbf{R}^{n}$. Since we are assuming that $G$ is of class $C^{2}$ and that $\left(\partial^{2} G(u) / \partial u_{i} \partial u_{j}\right)$ is uniformly bounded it follows that $J$ is of class $C^{2}$. We observe that if $J_{N}$ denotes the restriction of $J$ to $E_{N}$ then

$$
\left\langle\nabla J_{N}(u), v\right\rangle=\int_{\Omega}(\partial u / \partial x, \partial v / \partial x)-(\partial u / \partial t, \partial v / \partial t)+(\nabla G(u), v)-(f, v)
$$

for all $u, v \in E_{N}$, where $\left\langle,>\right.$ denotes the duality pairing. We denote by $D^{2} J_{N}(u)$ the Hessian of $J_{N}$ at $u$.

LEMma 2.2: For each positive integer $N$ there exists a unique $u_{N} \in E_{N}$ such that $\nabla J_{N}\left(u_{N}\right)=0$. Moreover,

$$
J_{N}\left(u_{N}\right)=\max _{x \in X_{N}} \min _{z \in Z_{N}} J(x+z) .
$$

Proof. Fix $u \in E_{N}$; then for each $w=\sum_{i, k, l} \mu_{i k l} \phi_{k l} a_{i} \in Z_{N}$

$$
\begin{aligned}
\left\langle D^{2} J_{N}(u) w, w\right\rangle & =\int_{\Omega}|\partial w / \partial x|^{2}-|\partial w / \partial t|^{2}+\left(\left(\partial^{2} G(u) / \partial u_{i} \partial u_{j}\right) w, w\right) \\
& \geqslant \int_{\Omega}|\partial w / \partial x|^{2}-|\partial w / \partial t|^{2}+(A w, w) \\
& =\sum_{i, k, l}\left(l^{2}-k^{2}+\alpha_{i}\right) \mu_{i k l}^{2} \geqslant m_{1}\|w\|_{0}^{2}
\end{aligned}
$$

where $m_{1}=\min \left\{l^{2}-k^{2}+\alpha_{i}: 1 \leqslant i \leqslant n, l, k \in \mathbf{N}, l^{2}-k^{2}+\alpha_{i}>0\right\}$. Similarly, for $v \in X_{N}$,

$$
\left\langle D^{2} J_{N}(u) v, v\right\rangle \leqslant \sum_{i, k, l}\left(l^{2}-k^{2}+\beta_{i}\right) \mu_{i k l}^{2} \leqslant-m_{2}\|v\|_{0}^{2}
$$


where $m_{2}=\min \left\{k^{2}-l^{2}-\beta_{i}: 1 \leqslant i \leqslant n, l, k \in \mathbf{N}, l^{2}-k^{2}+\beta_{i}<0\right\}$. From this we conclude that $X_{N} \cap Z_{N}=\{0\}$. It is easy to see that $\operatorname{dim} Z_{N}=\operatorname{dim} Y_{N}$ and so $E_{N}=X_{N} \oplus Z_{N}$. In addition, (2.4) and (2.5) show that the hypotheses of Lemma 2.1 are satisfied, which completes the proof.

We now write $u_{N}=u_{N}^{1}+u_{N}^{2}$ with $u_{N}^{1} \in X_{N}$ and $u_{N}^{2} \in Z_{N}$. By Lemma 2.2 we have

$$
\begin{aligned}
0 & =\left\langle\nabla J_{N}\left(u_{N}\right), u_{N}^{1}-u_{N}^{2}\right\rangle \\
& =\int_{\Omega}\left(\partial u_{N}^{1} / \partial x, \partial u_{N}^{1} / \partial x\right)-\left(\partial u_{N}^{1} / \partial t, \partial u_{N}^{1} / \partial t\right)-\left(\partial u_{N}^{2} / \partial x, \partial u_{N}^{2} / \partial x\right)+\left(\partial u_{N}^{2} / \partial t, \partial u_{N}^{2} / \partial t\right) \\
& +\left(\int_{0}^{1}\left(\partial^{2} G\left(s u_{N}\right) / \partial u_{i} \partial u_{j}\right) u_{N} \mathrm{~d} s,\left(u_{N}^{1}-u_{N}^{2}\right)\right)-\left(f, u_{N}^{1}-u_{N}^{2}\right) \\
& \leqslant \int_{\Omega}\left(\partial u_{N}^{1} / \partial x, \partial u_{N}^{1} / \partial x\right)-\left(\partial u_{N}^{1} / \partial t, \partial u_{N}^{1} / \partial t\right)-\left(\partial u_{N}^{2} / \partial x, \partial u_{N}^{2} / \partial x\right)+\left(\partial u_{N}^{2} / \partial t, \partial u_{N}^{2} / \partial t\right)+\left(B u_{N}^{1}, u_{N}^{1}\right) \\
& -\left(A u_{N}^{2}, u_{N}^{2}\right)+\|f\|_{0} \cdot\left(\left\|u_{N}^{1}\right\|_{0}+\left\|u_{N}^{2}\right\|_{0}\right) \\
& \leqslant-m_{1}\left\|u_{N}^{1}\right\|_{0}^{2}-m_{2}\left\|u_{N}^{2}\right\|_{0}^{2}+\|f\|_{0}\left(\left\|u_{N}^{1}\right\|_{0}+\left\|u_{N}^{2}\right\|_{0}\right)
\end{aligned}
$$

where $m_{1}$ and $m_{2}$ are as in Lemma 2.2. Since $m_{1}$ and $m_{2}$ are positive and independent of $N$ inequality (2.6) proves that $\left\{u_{N}\right\}_{N}$ is bounded in $\left(L_{2}(\Omega)\right)^{n}$.

It is well documented that for each $h \subset R(\square)$ there exists a unique $w$ in the orthogonal complement of $\operatorname{Ker}(\square)$ which is a weak solution of $\square u=h$ in $\Omega, u \equiv 0$ on $\partial \Omega$. Moreover, $w \in\left(H^{1}(\Omega)\right)^{n}$ and there exists a constant $c>0$ such that

$$
\|w\|_{1} \leqslant c\|h\|_{0} .
$$

Now we write $u_{N}=v_{N}+w_{N}$ with $v_{N} \in R(\square)$ and $w_{N} \in \operatorname{Ker}(\square)$. We let $Q_{N}$ denote the orthogonal projection onto $E_{N} \cap R(\square)$. Because of Lemma 2.2 we have $\square v_{N}=Q_{N}\left(f-\nabla G\left(u_{N}\right)\right)$ in $\Omega, v_{N} \equiv 0$ on $\partial \Omega$. Hence by (2.7), we have

$$
\begin{aligned}
\left\|v_{N}\right\|_{1} & \leqslant c\left(\|f\|_{0}+\left\|\int_{0}^{1}\left(\partial^{2} G\left(s u_{N}\right) / \partial u_{i} \partial u_{j}\right) u_{N} \mathrm{~d} s\right\|_{0}\right) \\
& \leqslant c\left(\|f\|_{0}+\sup \left\{\left|\alpha_{1}\right|\left\|u_{N}\right\|_{0},\left|\beta_{n}\right|\left\|u_{N}\right\|_{0} ; N=1,2, \ldots\right\}\right) \\
& \equiv \mathbf{K} .
\end{aligned}
$$

\section{PROOF OF THEOREM A}

First we claim that $\left\{u_{N}\right\}_{N}$ is bounded in $\left(\dot{H}^{1}(\Omega)\right)^{n}$. To show this, by (2.8) it is sufficient to prove that $\left\{w_{N}\right\}_{N}$ is bounded in $\left(\dot{H}^{1}(\Omega)\right)^{n}$. To do so we write $w_{N}=w_{N}^{+}+w_{N}^{-}$, where $w_{N}^{-} \in X_{N}$ and $w_{N}^{+} \in Z_{N}$. Since $v=\partial^{2} w_{N}^{+} / \partial t^{2}-\partial^{2} w_{N}^{-} / \partial t^{2} \in E_{N} \cap \operatorname{Ker}(\square)$, by Lemma 2.2 we have

$$
0=\int_{\Omega}\left(\nabla G\left(u_{N}\right), v\right)-(f, v) .
$$

Therefore, integrating by parts, using (1.2) and $\nabla G(0)=0$ and noting that $\left\|\partial w_{N} / \partial t\right\|_{0}=\left\|\partial w_{N} / \partial x\right\|_{0}$ 
we have

$$
\begin{aligned}
\|\partial f / \partial t\|_{0}\left(\left\|w_{N}^{+}\right\|_{1}+\left\|w_{N}^{-}\right\|_{1}\right) & \geqslant \int_{\Omega}\left(\left(\partial^{2} G\left(u_{N}\right) / \partial u_{i} \partial u_{j}\right)\left(\partial u_{N} / \partial t\right), \partial w_{N}^{+} / \partial t-\partial w_{N}^{-} / \partial t\right) \\
& \geqslant-C_{1}\left\|w_{N}\right\|_{1}+\int_{\Omega}\left(A \partial w_{N}^{+} / \partial t, \partial w_{N}^{+} / \partial t\right)-\int_{\Omega}\left(B \partial w_{N}^{-} / \partial t, \partial w_{N}^{-} / \partial t\right) \\
& \geqslant-C_{1}\left\|w_{N}\right\|_{1}+C_{2}\left\|w_{N}^{+}\right\|_{1}^{2}+C_{3}\left\|w_{N}^{-}\right\|_{1}^{2}
\end{aligned}
$$

where $C_{1}$ comes from the facts that $\left(\partial^{2} G(u) / \partial u_{i} \partial u_{j}\right)$ and $\left\{\left\|v_{N}\right\|_{1}\right\}_{N}$ are uniformly bounded, $2 C_{2}=\min \left\{\alpha_{i} ; \alpha_{i}>0\right\}$ and $2 C_{3}=\min \left\{-\beta_{i} ; \beta_{i}<0\right\}$. From (3.2) it is clear that $\left\{\left\|u_{N}\right\|_{1}\right\}$ is bounded. Let $\left(v_{0}, w_{0}\right) \in\left(\dot{H}^{1}(\Omega)\right)^{n} \times\left(H^{1}(\Omega)\right)^{n}$ be such that some subsequence $\left\{\left(v_{N_{j}}, w_{N_{j}}\right)\right\}_{j}$ converges weakly in $\left(H^{1}(\Omega)\right)^{n} \times\left(H^{1}(\Omega)\right)^{n}$ to $\left(v_{0}, w_{0}\right)$.

We claim that $u_{0}=v_{0}+w_{0}$ is a weak solution of (1.1). Let $\phi: \Omega \rightarrow \mathbf{R}^{n}$ be any $C^{\infty}$ function with compact support in $\Omega$. Let $\phi_{j}$ be the orthogonal projection of $\phi$ on $E_{N_{j}}$. Since $\bigcup_{j=1}^{\infty} E_{N_{j}}$ is dense in $\left(L_{2}(\Omega)\right)^{n}$ we see that $\phi_{j} \rightarrow \phi$ in $\left(L_{2}(\Omega)\right)^{n}$ and $\square \phi_{j} \rightarrow \square \phi$ in $\left(L_{2}(\Omega)\right)^{n}$. Therefore, using Lemma 2.2 we have

$$
\begin{aligned}
& \int_{\Omega}\left(v_{0}, \square \phi\right)+\left(\nabla G\left(u_{0}\right), \phi\right)-(f, \phi) \\
& =\int_{\Omega}\left(v_{0}, \square\left(\phi-\phi_{j}\right)\right)+\left(\nabla G\left(u_{0}\right), \phi-\phi_{j}\right)-\left(f, \phi-\phi_{j}\right) \\
& +\left(\nabla G\left(u_{0}\right)-\nabla G\left(v_{N_{j}}+w_{N_{j}}\right), \phi_{j}\right)+\left(v_{0}-v_{N_{j}}, \sqcup \phi_{j}\right) .
\end{aligned}
$$

It is clear that the right-hand side of (3.3) tends to zero as $j$ tends to infinity. Hence $u_{0}$ is a weak solution of $(1.1)$ which by construction is in $\left(\stackrel{H}{H}^{1}(\Omega)\right)^{n}$ and this proves the existence part of Theorem A.

Finally, we prove that (1.1) has at most one weak solution. Suppose that $u^{1}$ and $u^{2}$ are two such solutions. For $i=1$, 2, let $u_{N}^{i}=x_{N}^{i}+z_{N}^{i}$ be the projection of $u^{i}$ onto $E_{N}$, where $x_{N}^{i} \in X_{N}$ and $z_{N}^{i} \in Z_{N}$. Let $v_{N}=x_{N}^{1}-x_{N}^{2}, w_{N}=z_{N}^{1}-z_{N}^{2}$. We have

$$
\begin{aligned}
& 0=\int_{\Omega}\left\{\left(u^{1}-u^{2}, \square\left(v_{N}-w_{N}\right)\right)+\left(\int_{0}^{1}\left(\partial^{2} G\left(u^{2}+s\left(u^{1}-u^{2}\right)\right) / \partial u_{i} \partial u_{j}\right)\left(u^{1}-u^{2}\right) \mathrm{d} s, v_{N}-w_{N}\right)\right\} \\
& =\int_{\Omega}\left\{\left(v_{N}+w_{N}, \square\left(v_{N}-w_{N}\right)\right)+\left(\int_{0}^{1}\left(\partial^{2} G\left(u^{2}+s\left(u^{1}-u^{2}\right)\right) / \partial u_{i} \partial u_{j}\right)\left(v_{N}+w_{N}\right) \mathrm{d} s, v_{N}-w_{N}\right)\right. \\
& +\left(\int_{0}^{1}\left(\partial^{2} G\left(u^{2}+s\left(u^{1}-u^{2}\right)\right) / \partial u_{i} \partial u_{j}\right)\right. \\
& \left.\left.\left(u^{1}-u_{N}^{1}+u_{N}^{2}-u^{2}\right) \mathrm{d} s, v_{N}-w_{N}\right)\right\} \\
& \leqslant \int_{\Omega}\left\{\left(\square v_{N}+B v_{N}, v_{N}\right)-\left(\square w_{N}+A w_{N}, w_{N}\right)\right\}+C\left(\left\|u^{1}-u_{N}^{1}\right\|_{0}+\left\|u^{2}-u_{N}^{2}\right\|_{0}\right) \\
& \leqslant-m_{2}\left\|v_{N}\right\|_{0}^{2}-m_{1}\left\|w_{N}\right\|_{0}^{2}+C\left(\left\|u^{1}-u_{N}^{1}\right\|_{0}+\left\|u^{2}-u_{N}^{2}\right\|_{0}\right) \text {. }
\end{aligned}
$$

Consequently, $v_{N}$ and $w_{N}$ tend to zero as $N \rightarrow \infty$, and so $u^{1}=u^{2}$. 


\section{REFERENCES}

1. Ahmad S., An existence theorem for periodically perturbed conservative systems, Mich Math. J. 20, 385 392 (1973).

2. LAZER A. C., Application of a lemma on bilinear forms to a problem in nonlinear oscillations, Proc. Am. math. Soc. 33, 311327 (1970).

3. Lazer A. C., Landesman E. M. \& Meyers D. R., On saddle point problems in the calculus of variations, the Ritz algorithm, and monotone convergence, J. math. Analysis Applic. 52, 594-614 (1975).

4. MAWHIN J., Solutions périodiques d'equations aux dérivées partielles hyperboliques nonlinéares, pp. 301-315, Mélanges Th. Vogel, Presses Univ. de Bruxelles (1978). 